\title{
Ultrasound-Guided Left Stellate Ganglion Blocks for Recurrent Ventricular Tachycardia (Electrical Storm)
}

\author{
Michael R. Ander*, Scott W. Byram, Ulana B. Sonevytsky \\ Department of Anesthesiology, Loyola University Medical Center, Maywood, IL, USA \\ Email: *miander@lumc.edu, sbyram@lumc.edu,ubdzwinyk@gmail.com
}

How to cite this paper: Ander, M.R., Byram, S.W. and Sonevytsky, U.B. (2018) Ultrasound-Guided Left Stellate Ganglion Blocks for Recurrent Ventricular Tachycardia (Electrical Storm). Open Journal of Anesthesiology, 8, 80-84.

https://doi.org/10.4236/ojanes.2018.83008

Received: January 19, 2018

Accepted: March 18, 2018

Published: March 21, 2018

Copyright $\odot 2018$ by authors and Scientific Research Publishing Inc. This work is licensed under the Creative Commons Attribution International License (CC BY 4.0).

http://creativecommons.org/licenses/by/4.0/

\begin{abstract}
Recurrent Ventricular Tachycardia, or Electrical Storm, is a relatively common cause of mortality and morbidity after myocardial infarction. We present a case in which left stellate ganglion blocks were performed under ultrasound guidance in order to alleviate recurrent ventricular tachycardia in a patient requiring extracorpeal membrane oxygenation after myocardial infarction. A medically complex 54-year-old male after two weeks removed from an acute ST elevation myocardial infarction status post stenting and intra-aortic balloon pump placement presented in the cardiovascular intensive care unit with recurrent unstable ventricular tachycardia while on extracorporeal membrane oxygenation and multiple vasoactive infusions. Our acute pain service was consulted by the cardiac electrophysiology service to provide left stellate ganglion blocks in attempt to attenuate electrical storm. Multiple single shot left stellate ganglion blocks were performed on subsequent days with increasing local anesthetic concentrations and volumes successfully providing temporary relief of electrical storm. A left stellate ganglion catheter was eventually placed, and a continuous infusion of local anesthesia was started. The patient experienced complete relief from unstable ventricular tachycardia and no further defibrillations were required. The catheter remained in place for 14 days allowing for extracorporeal membrane oxygenation decannulation without the return of electrical storm. We believe ultrasound guided left stellate ganglion blocks to be a relatively safe means to provide temporary relief of recurrent ventricular tachycardia.
\end{abstract}

\section{Keywords}

Stellate Ganglion Block, Electrical Storm, Recurrent Ventricular Tachycardia 


\section{Introduction}

Recurrent Ventricular Tachycardia (RVT), or Electrical Storm (ES), is a relatively common cause of mortality and morbidity after myocardial infarction [1] [2]. ES is defined as three or more sustained episodes of ventricular tachycardia (VT) or ventricular fibrillation requiring shocks from a defibrillator within a 24-hour period [1]. These episodes must last greater than 30 seconds in duration, involve hemodynamic compromise, and require intervention to terminate [1]. We present a case in which left stellate ganglion blocks (SGBs) were performed under ultrasound guidance to alleviate ES in a patient requiring extracorporeal membrane oxygenation (ECMO) after myocardial infarction.

\section{Case Report}

A medically complex 54-year-old male two weeks removed from an anterior ST-elevation myocardial infarction (STEMI) status post drug eluting stent to the proximal left anterior descending coronary artery and intra-aortic balloon pump placement (IABP) presented in the cardiovascular ICU with recurrent stable VT initially controlled by amiodarone and lidocaine infusions. The lidocaine infusion was discontinued on post-initiation day 1 after the patient sustained a seizure. His condition further deteriorated when he progressed into sustained, hemodynamically unstable RVT which required more than twenty defibrillations in a 24 -hour period, multiple vasoactive infusions, multiple antiarrhythmic infusions, and the institution of ECMO.

Our Acute Pain Service was consulted by the Cardiac Electrophysiology (EP) Service to provide left SGBs in attempt to attenuate electrical storm. Multiple single shot left SGBs were performed on subsequent days with increasing local anesthetic concentrations and volumes successfully providing temporary relief of electrical storm. A left stellate ganglion catheter was eventually placed, and a continuous infusion of local anesthesia was started. The patient experienced complete relief from unstable VT and no further defibrillations were required. The catheter remained in place for 14 days allowing for ECMO decannulation without the return of electrical storm; however, the patient was unable to be weaned off mechanical ventilation, IABP, or vasopressors due to acute ischemic cardiomyopathy. The patient's family withdrew care shortly thereafter, and the patient expired. The discussed patient's wife (next-of-kin) approved the reporting of this case.

\section{Discussions}

The SGB, more appropriately called the cervical sympathetic trunk (CST), is a well-established intervention for sympathetically mediated disorders including cardiac arrhythmias, vascular insufficiencies, and multiple pain syndromes [1]

[2] [3].

The cervical stellate ganglion (CSG) is present in approximately $80 \%$ of the population and is located just anterior to the transverse process of the $\mathrm{C} 7$ verte- 
bra, superior to the first rib. Along with the middle cervical ganglion, traversing fibers of the CSG are present at the level of C6, and are located anterolaterally to the belly of the longus colli muscle (Figure 1) [3]. Even in the setting of a normal heart, predominance of left ventricular innervation by either the right stellate ganglion (RSG) or left stellate ganglion (LSG) is not well understood and remains controversial [4] [5]. It appears that both RSG and LSG provide variable degrees of sympathetic cardiac innervation [4] [5] [6]. Ultimately, left SGB is superior to right SGB in terminating ventricular arrhythmias and thus, we choose to perform left SGBs [4] [5].

Although traditionally described as a blind technique, the use of ultrasound guidance has resulted in lower volume of local anesthesia requirements, fewer hematomas creations and a more rapid onset of Horner Syndrome. Described complications of SGB include unintentional esophageal and tracheal puncture, injury to the pleura and lung, recurrent laryngeal and phrenic nerve injury, thyroid gland trauma, infectious complications, epidural and intrathecal spread, intravascular injection of the carotid artery, jugular veins, or vertebral artery leading to seizure and cardiac arrest [1] [7] [8]. Contraindications of the procedure include anticoagulation, patient refusal, infection at the site of injection, and bilateral block [1].

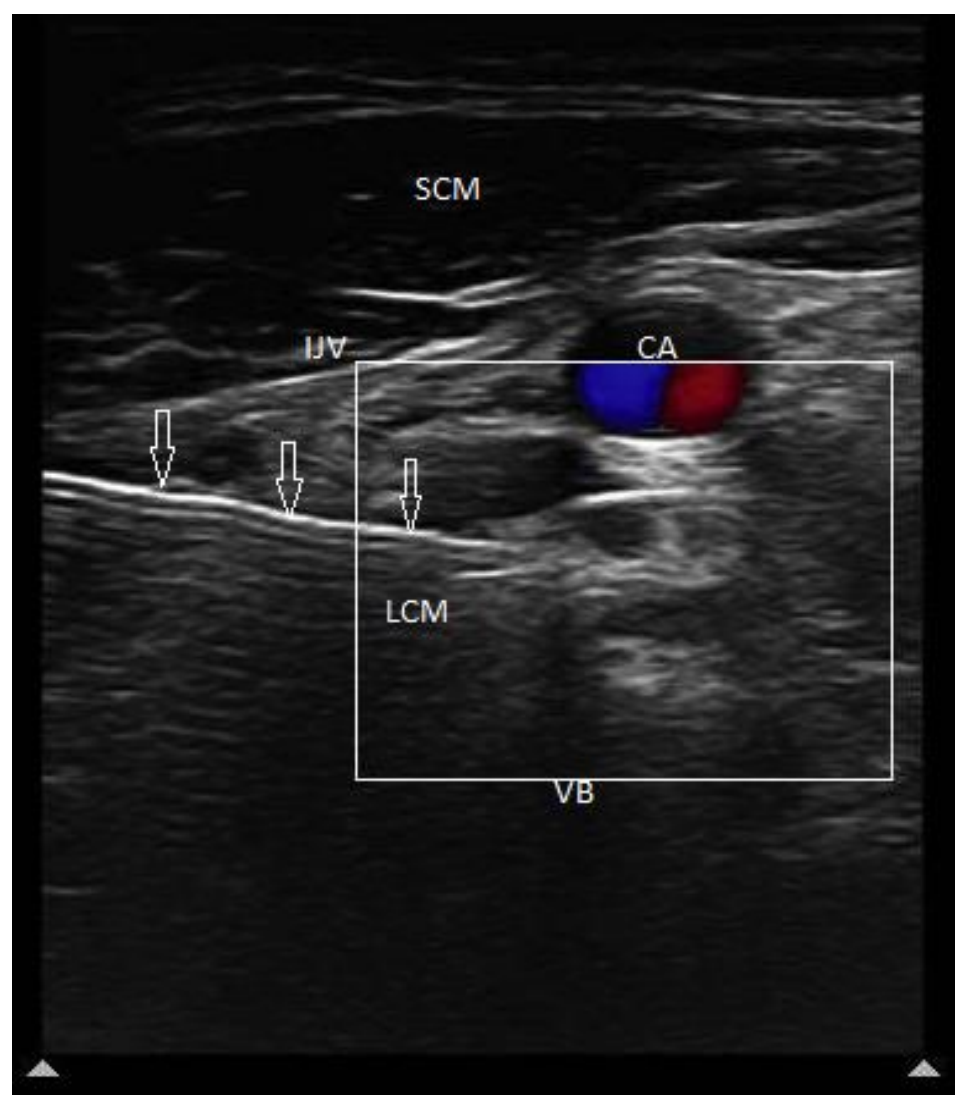

Figure 1. Block needle (arrowheads). VB, C6 vertebral body; LCM, longus colli muscle; IJV, internal jugular vein; CA, carotid artery; SCM, sternocleidomastoid muscle. 
Given the risk for inadvertent vascular puncture and potential subsequent hematoma formation, anticoagulation is a commonly listed contraindication of ultrasound-guided SGB. Our patient was fully anticoagulated at the time we performed our procedures. The decision to proceed with the block was made as the procedures were palliative in nature, which was clearly understood by the patient's family members and all inter-disciplinary teams involved in this patient's care. EP was consulted for sustained VT, but ablation was not possible in the patient due to hemodynamic instability and the requirements of ECMO and vasoactive infusions. As our cardiac electrophysiology service suspected our patient's electrical storm was catecholamine dependent, providing a partial sympathectomy via left SGBs allowed temporary cessation of sustained unstable refractory VT.

ES is an important cause of morbidity and mortality in patients with recent ischemic cardiac injury. The increased use of ultrasound guidance allows SGBs to be performed with greater precision and, thus, allows for reliable success with fewer complications. We believe ultrasound guided left SGBs to be a relatively safe means to provide temporary relief of RVT.

\section{Funding}

This research did not receive any specific grant from funding agencies in the public, commercial, or not-for-profit sectors.

\section{Conflicts of Interest}

None.

\section{References}

[1] Eifling, M., Razavi, M. and Massumi, A. (2011) The Evaluation and Management of Electrical Storm. Texas Heart Institute Journal, 38, 111.

[2] Stevenson, W.G., et al. (2008) Irrigated Radiofrequency Catheter Ablation Guided by Electroanatomic Mapping for Recurrent Ventricular Tachycardia after Myocardial Infarction the Multicenter Thermocool Ventricular Tachycardia Ablation Trial. Circulation, 118, 2773-2782. https://doi.org/10.1161/CIRCULATIONAHA.108.788604

[3] Gofeld, M., et al. (2009) Development and Validation of a New Technique for Ultrasound-Guided Stellate Ganglion Block. Regional Anesthesia and Pain Medicine, 34, 475-479. https://doi.org/10.1097/AAP.0b013e3181b494de

[4] Scanlon, M., et al. (2015) Urgent Ultrasound-Guided Bilateral Stellate Ganglion Blocks in a Patient with Medically Refractory Ventricular Arrhythmias. Critical Care Medicine, 43, e316-e318. https://doi.org/10.1097/CCM.0000000000001086

[5] Vaseghi, M., et al. (2012) Sympathetic Innervation of the Anterior Left Ventricular Wall by the Right and Left Stellate Ganglia. Heart Rhythm, 9, 1303-1309. https://doi.org/10.1016/j.hrthm.2012.03.052

[6] Yanowitz, F., et al. (1966) Functional Distribution of Right and Left Stellate Innervation to the Ventricles: Production of Neurogenic Electrocardiographic Changes by Unilateral Alteration of Sympathetic Tone. Circulation Research, 18, 416-428. 
https://doi.org/10.1161/01.RES.18.4.416

[7] Elias, M. (2000) Cervical Sympathetic and Stellate Ganglion Blocks. Pain Physician, 3, 294-304.

[8] Rastogi, S. and Tripathi, S. (2010) Cardiac Arrest Following Stellate Ganglion Block Performed under Ultrasound Guidance. Anaesthesia, 65, 1042.

https://doi.org/10.1111/j.1365-2044.2010.06487.x 